\title{
Antibodies to human gastric epithelial cells and heat shock protein 60 in Helicobacter pylori positive mucosa associated lymphoid tissue lymphoma
}

\author{
Y Kawahara, K Yokota, M Mizuno, N Yunoki, T Uesu, H Okada, K Kobayashi, Y Hirai, \\ K Oguma, T Tsuji
}

\begin{abstract}
Background-Development of gastric mucosa associated lymphoid tissue (MALT) lymphoma is thought to be closely associated with host immune reactions to Helicobacter pylori.

Aim-To investigate humoral immune responses in patients with MALT lymphoma to antigens shared by $H$ pylori and human gastric epithelial cells.

Methods-Sera were obtained from $H$ pylori positive patients with MALT lymphoma $(n=11)$ or other gastroduodenal diseases (peptic ulcer, $n=40$; nonulcer dyspepsia, $\mathrm{n}=20$ ) and from $\mathrm{H}$ pylori negative healthy control subjects $(n=10)$. Antibodies to HGC-27 human gastric epithelial cells and human recombinant heat shock protein (Hsp) 60 were examined using an enzyme linked immunosorbent assay (ELISA) and immunoblotting.

Results-Antibody titres to HGC-27 cells were significantly elevated in $H$ pylori positive patients with MALT lymphoma when compared with titres in patients with other gastroduodenal diseases and in healthy subjects. Immunoblotting of sera from patients with MALT lymphoma often detected a band with a molecular mass corresponding to Hsp60, and both ELISA and immunoblotting showed elevated antibody titres to the recombinant human Hsp60. Antigenic similarity between Hsp60 and $H$ pylori HspB was documented by immunoblotting experiments. Conclusions-Autoantibodies reactive with host gastric epithelial cells are often increased in MALT lymphoma, and Hsp60 is a major target antigen. Immune responses induced by immunological cross reactivity between $H$ pylori $\mathrm{HspB}$ and human Hsp60 in gastric epithelium may be involved in the development of MALT lymphoma.

(Gut 1999;45:20-23)
\end{abstract}

Keywords: Helicobacter pylori; mucosa associated lymphoid tissue lymphoma; heat shock protein; autoantibody

Accumulating evidence suggests that Helicobacter pylori is involved in the pathogenesis of gastric lymphoma of mucosa associated lymphoid tissue (MALT) type. ${ }^{1-4}$ Antigenic mimicry between $H$ pylori and the host mucosa ${ }^{5}$ may induce host autoimmune responses which lead to the development of the disease. Heat shock protein ( $\mathrm{Hsp}$ ) is one candidate for the cross reacting antigen(s). ${ }^{6}$ Hsps are a family of proteins induced by cell stress, such as microbial infections, and are conserved in both prokaryotic and eukaryotic cells. Hsps with identical antigenic structures have been shown by immunohistochemistry in $H$ pylori bacterial cells and gastric epithelial cells of patients infected with $\mathrm{H}$ pylori. ${ }^{7}$ In this study, we investigated host humoral immune responses to $H$ pylori which may lead to the production of autoreactive antibodies. We measured antibodies against gastric epithelial cells and human Hsp60 in patients with MALT lymphoma, peptic ulcers, and non-ulcer dyspepsia using an enzyme linked immunosorbent assay (ELISA) and immunoblotting.

\section{Patients and methods}

PATIENTS

Sera were obtained from $71 \mathrm{H}$ pylori positive patients, including 11 with MALT lymphoma (six women and five men; mean age 59.8 years), 20 with gastric ulcer (three women and 17 men; mean age 46.3 years), 20 with duodenal ulcer (10 women and 10 men; mean age 30.4 years), and 20 with non-ulcer dyspepsia (six women and 14 men; mean age 41.5 years). Sera were also obtained from 10 healthy volunteers (seven women and three men; mean age 37.9 years old). Informed consent was obtained from each patient and healthy volunteer.

Diagnosis was made based on findings of endoscopic examination and histological examination of gastric biopsy or gastrectomy specimens. Histology of MALT lymphoma was assessed according to the REAL classification. ${ }^{8}$ Tissue specimens were analysed immunohistochemically using antibodies to $\operatorname{Ig} \kappa, \operatorname{Ig} \lambda, \operatorname{CD} 20$, CD3, CD75, and bcl-2 (Dako Japan, Tokyo, Japan). Immunoglobulin heavy chain gene rearrangement was examined by Southern blotting and hybridisation with a probe to the joining region of the immunoglobulin heavy chain gene. ${ }^{9}$ All the lymphomas showed lymphoepithelial lesions histologically and an immunophenotype compatible with that of MALT lymphoma. Monoclonality of the tumours was shown either by light chain restriction immunohistochemically or by molecular

Abbreviations used in this paper: MALT, mucosa associated lymphoid tissue; Hsp, heat shock protein; ELISA, enzyme linked immunosorbent assay; PBS, phosphate buffered saline. 


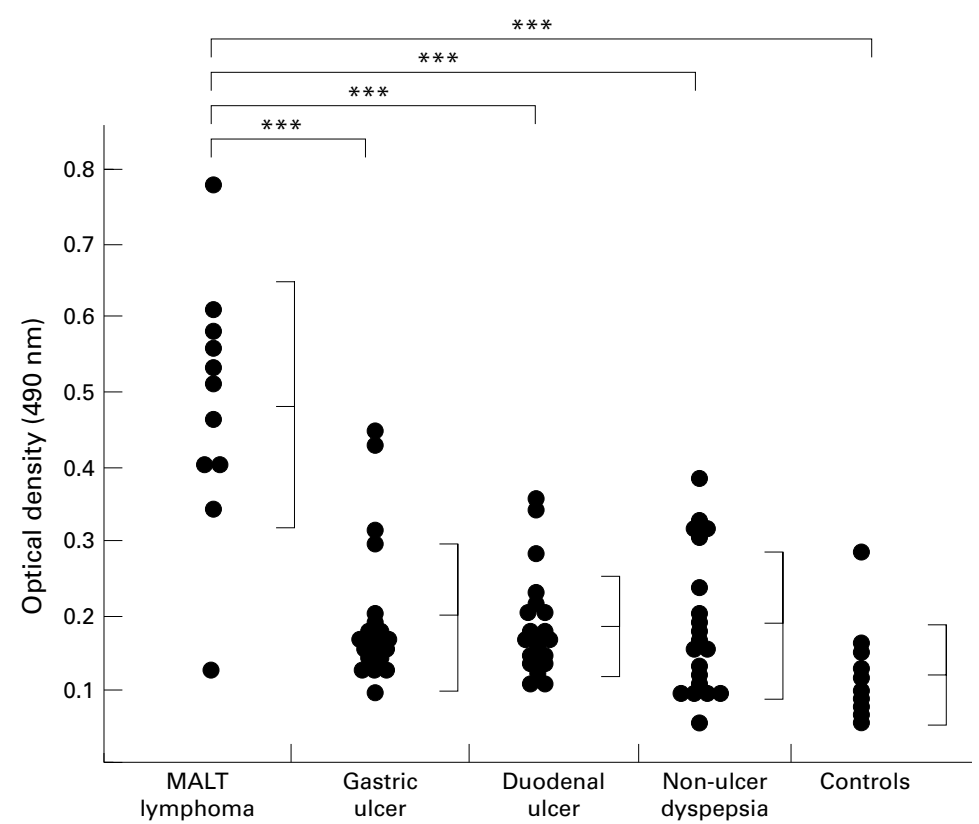

Figure 1 Serum antibody titres to HGC-27 cells in patients with mucosa associated lymphoid tissue mucosa associated lymphoid tissue (MALT) lymphoma, gastric ulcer, duodenal ulcer, or non-ulcer dyspepsia, and control healthy subjects. Serum antibodies to HGC-27 human gastric epithelial cells were measured using cell ELISA with plates seeded with HGC-27 cells. ${ }^{\star * *} p<0.001$ (Student's $t$ test).

genetic examination. All the lymphomas were low grade.

Infection with $H$ pylori was documented by culture, rapid urease test, and histology of gastric biopsy specimens and by the presence of serum antibodies to $H$ pylori by ELISA. ${ }^{10} H$ pylori was detected in the gastric mucosa of all patients whose sera were positive for anti- $H$ pylor $i$ antibodies. Negative $H$ pylor $i$ infection of the control healthy volunteers was defined by seronegativity to $H$ pylori.

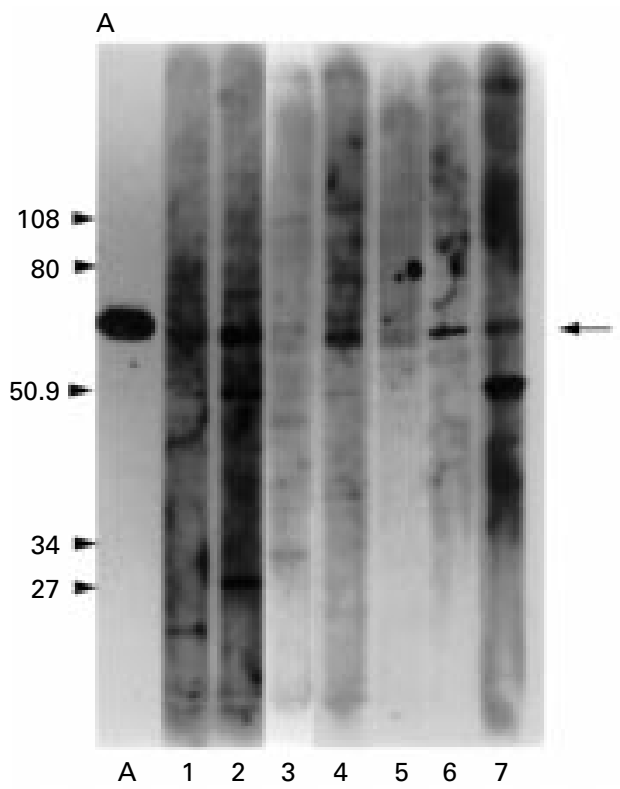

ELISA FOR THE DETECTION OF ANTIBODIES TO HUMAN GASTRIC EPITHELIAL CELLS AND HUMAN HSP60

Serum antibodies to gastric epithelial cells were measured by cell ELISA. HGC-27 human gastric cancer cells ${ }^{11}$ were cultured in 96-well microtitre plates. The cells were washed once gently with phosphate buffered saline (PBS), fixed with $2 \%$ formalin in PBS for two hours at room temperature, and washed twice with PBS. After blocking with PBS containing 10\% skimmed milk, plates were incubated with 1:100 diluted serum for two hours, and washed with PBS containing $0.05 \%$ Tween 20 . Then peroxidase labelled rabbit anti-human IgG antibody (Dako Japan) was added, and the plates were incubated for two hours. After being washed, the well contents were allowed to react with $o$-phenylenediamine in citrate buffer, pH 5.5. Absorbance at $490 \mathrm{~nm}$ was measured by an ELISA plate reader.

For the detection of antibodies to human Hsp60, wells of microtitre plates were coated with a recombinant human Hsp60 protein (Stress Gen, Victoria, British Columbia, Canada) (10 mg protein/well) and blocked with PBS containing $10 \%$ skimmed milk. The plates were incubated with 1:100 diluted serum for two hours at room temperature and washed with PBS containing $0.05 \%$ Tween 20, and bound human antibodies were detected as described above.

\section{IMMUNOBLOTTING}

Sonicated $H$ pylori extracts, HGC-27 cells, and the recombinant human Hsp60 were dissolved in $1 \%$ sodium dodecyl sulphate containing 5\% 2-mercaptoethanol and separated by sodium dodecyl sulphate/polyacrylamide gel electrophoresis $(10 \%$ gel). The separated proteins

B

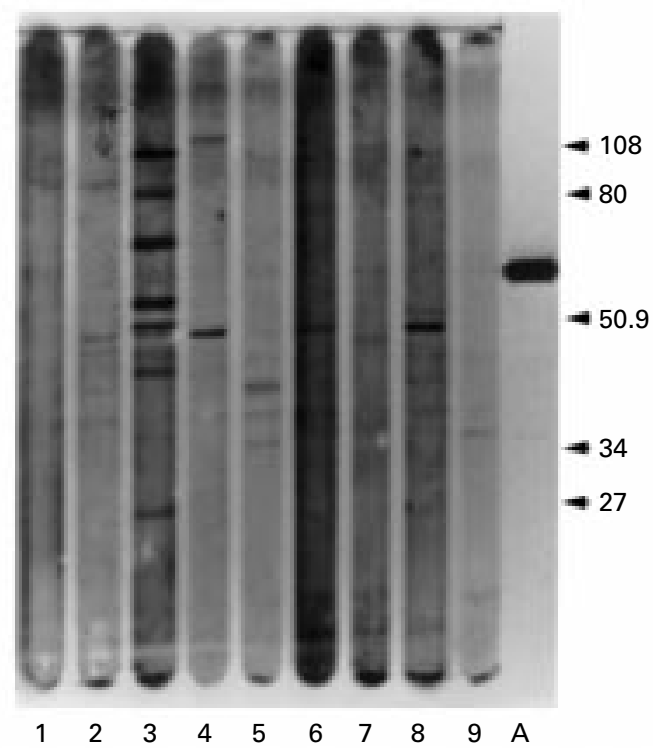

Figure 2 Immunoblots of $H G C-27$ cells allowed to react with serum from various patient and control groups. Lysates of HGC-27 cells were fractionated on $10 \%$ polyacrylamide gels and then immunoblotted. (A) Lane A, The membrane strip was allowed to react with LK-2 mouse monoclonal antibody to human Hsp60. Lanes 1-7, the strips were allowed to react with serum from patients with mucosa associated lymphoid tissue (MALT) lymphoma. Bands with an approximate molecular mass of $60 \mathrm{kDa}$ which correspond to Hsp60 are indicated by an arrow. (B) The membrane strip was allowed to react with serum from patients with non-ulcer dyspepsia (lanes 1-3), gastric ulcer (lanes 4-6), or duodenal ulcer (lanes 7 and 8), and from a healthy control subject (lane 9). Lane A, LK-2. 
Figure 3 Immunoblots of recombinant human Hsp60 from patients with mucosa associated lymphoid tissue (MALT) lymphoma. Hsp60 proteins were fractionated on $10 \%$ polyacrylamide gels and then immunoblotted. Lane $A$, the membrane strip was mouse monoclonal antibody to human Hsp60. Lanes 1-11, the strips were allowed to react with sera from patients with MALT lymphoma. Bands with approximate molecular mass $60 \mathrm{kDa}$, which correspond to Hsp60, are present in lanes $1-10$ (arrow). allowed to react with sera Recombinant human allowed to react with $\mathrm{LK}-2$

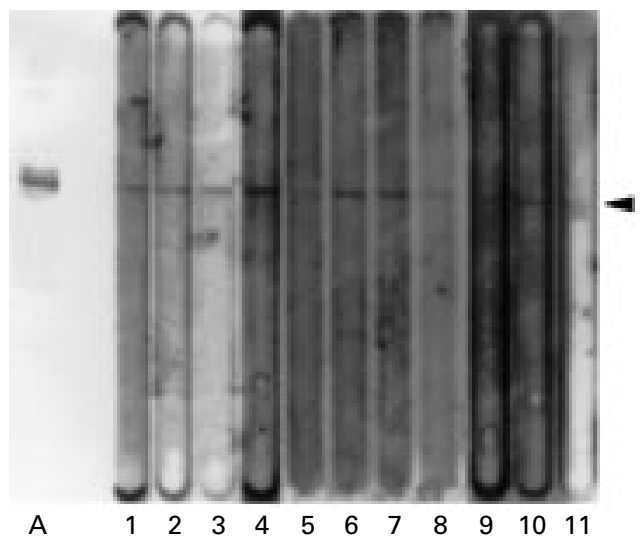

Table 1 Detection of human recombinant Hsp60 by immunoblotting

\begin{tabular}{llc}
\hline & $\begin{array}{l}\text { No of } \\
\text { patients }\end{array}$ & $\begin{array}{l}\text { Hsp60 } \\
\text { positivity }\end{array}$ \\
\hline MALT lymphoma & 11 & $10(91)^{\star}$ \\
Gastric ulcer & 20 & $3(15)$ \\
Duodenal ulcer & 20 & $3(15)$ \\
Non-ulcer dyspepsia & 20 & $4(20)$ \\
Healthy control & 10 & $0(0)$
\end{tabular}

Results in parentheses are percentages.

MALT, mucosa associated lymphoid tissue.

${ }^{\star} \mathrm{p}<0.01 v$ the other patient groups and healthy controls $\left(\chi^{2}\right.$ test $)$.

were electrophoretically transferred to polyvinylidene difluoride membrane (Millipore Japan, Tokyo, Japan). The membrane was cut into strips, and each strip was allowed to react with a patient's serum. After being washed with PBS containing $0.05 \%$ Tween 20 , the strips were allowed to react with peroxidase conjugated rabbit anti-human IgG antibody, and the reaction products were detected using an ECL western blotting detection system (Amersham International, Little Chalfont, Bucks, UK).

Cross reactivity of human $\mathrm{Hsp} 60$ and components of $H$ pylori was examined by immunoblotting with mouse monoclonal anti-

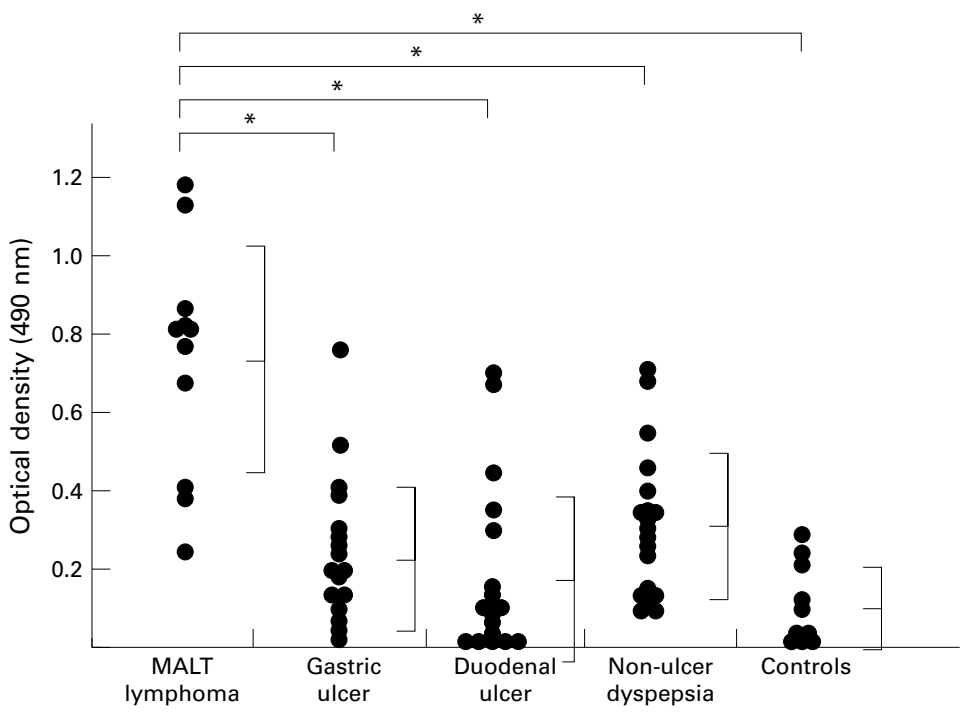

Figure 4 Serum antibody titres to recombinant human Hsp60 in patients with mucosa associated lymphoid tissue (MALT) lymphoma, gastric ulcer, duodenal ulcer, or non-ulcer dyspepsia, and control healthy subjects. Serum antibodies to human Hsp60 were measured using ELISA with plates coated with recombinant human Hsp60. ${ }^{\star} p<0.001$ (Student's $t$ test). body to Hsp60 (LK-2; Stress Gen). The membrane strips with proteins of $H$ pylori extracts, HGC-27 cells, and the human Hsp60 were prepared as described above and allowed to react with LK-2. After being washed, the strips were allowed to react with peroxidase conjugated swine anti-mouse IgG antibody (Dako) and developed using the chemiluminescence based detection kit as described above.

STATISTICAL ANALYSIS

Student's $t$ test and the $\chi^{2}$ test were used for statistical analyses.

\section{Results}

ANTIBODIES TO HUMAN GASTRIC CELLS

Figure 1 shows the distribution of antibody titres to HGC-27 human gastric epithelial cells in each group. Autoantibodies to HGC-27 cells were present in the serum of most patients with MALT lymphoma, and the antibody titres in these patients were significantly higher than those in the other patient groups and the healthy subjects $(p<0.001)$.

To define the target molecule(s) of the autoantibodies to HGC-27 cells, we performed immunoblotting using HGC-27 cells (fig 2). LK-2 anti-human Hsp60 mouse monoclonal antibody detected a band of molecular mass about $60 \mathrm{kDa}$, suggesting that HGC-27 cells constitutively express Hsp60. Serum from patients with MALT lymphoma detected various bands, and the band of molecular mass corresponding to Hsp60 was commonly observed in patients with MALT lymphoma (fig 2A). In contrast, serum from the other patient groups and the healthy control group rarely detected a band corresponding to Hsp60 (fig 2B).

Next, we tried to establish that the patients with MALT lymphoma had circulating antibodies to Hsp60, using immunoblotting and ELISA with the human recombinant Hsp60. On immunoblotting, 10 of 11 sera (91\%) from patients with MALT lymphoma recognised a band of the recombinant human Hsp60 (fig 3), whereas sera from the other patient groups and the healthy control detected it significantly less often $(0-20 \%, \mathrm{p}<0.01)$ (table 1). On ELISA, sera from patients with MALT lymphoma showed significantly higher antibody titres to the human Hsp60 than sera from the patients without MALT lymphoma and from the healthy control group $(\mathrm{p}<0.001)$ (fig 4$)$.

CROSS REACTIVITY OF LK2 ANTI-HUMAN HSP60 ANTIBODY TO $H$ PYLORI

LK-2 monoclonal antibody to the human Hsp60 recognised bands of $60 \mathrm{kDa}$ in both $H$ pylori and HGC-27 cells, which probably correspond to $H$ pylori $\mathrm{HspB}^{12-14}$ and human Hsp60 respectively (fig 5).

\section{Discussion}

In this study, we found that serum antibody titres to HGC-27 human gastric epithelial cell were significantly elevated in $H$ pylori positive patients with MALT lymphoma when compared with titres in $H$ pylori positive patients who had other gastroduodenal diseases and in healthy subjects without $H$ pylori infection. Our 


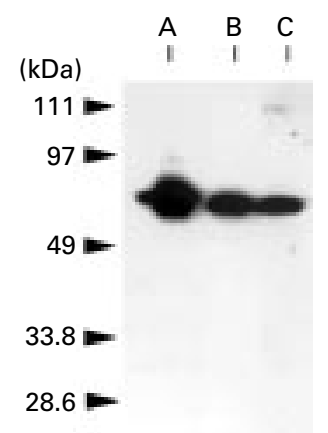

Figure 5 Immunoblots with LK-2 mouse monoclonal antibody to human Hsp60.

Recombinant human HSP60 (lane A), lysates of HGC-27 cells (lane B) and sonicates of $H$ pylori (lane $C$ ) were fractionated on $10 \%$ polyacrylamide gels and then immunoblotted. The membrane strip was allowed to react with $L K-2$ mouse monoclonal antibody to human Hsp60. Bands with approximate molecular mass $60 \mathrm{kDa}$ (Hsp60 in lane $A$ ) are also present in lanes $B$ and $C$. immunoblot experiments showed that sera from patients with MALT lymphoma often detected a band with a molecular mass corresponding to that of Hsp60. Moreover elevated antibody titres to the recombinant human Hsp60 were shown by ELISA and immunoblotting. Thus Hsp60 is probably a major antigen recognised by the anti-HGC-27 antibodies present in serum of patients with MALT lymphoma.

The LK-2 anti-human Hsp60 antibody recognises the epitope encoded by amino acid residues 383 to 419 of the human Hsp60 protein. ${ }^{15}$ We found that this antibody also detected a band corresponding to bacterial $\mathrm{HspB}$ in $H$ pylori extracts, suggesting that a common epitope(s) is present in human Hsp60 and its bacterial homologue, HspB. Thus infection of $H$ pylori may induce antibodies against bacterial $\mathrm{HspB}$ which cross react with a host Hsp60 through the molecular mimicry of these proteins, ${ }^{12}$ leading to the elevation of antibodies to HGC-27 cells in patients with MALT lymphoma. We have recently observed staining with LK-2 anti-Hsp60 antibody in follicular dendritic cells in germinal centres of gastric mucosa from patients with MALT lymphoma,${ }^{16}$ suggesting that the antigen recognised by the anti-Hsp60 antibody which originates from either the bacteria or the host cell is presented to immune cells in this disease.

We found that the immune response to Hsp60 was closely associated with MALT lymphoma. Although the association of MALT lymphoma with $H$ pylori strains expressing the CagA protein has been reported, ${ }^{17}$ no specific differences in $\mathrm{HspB}$ protein amino acid sequences between bacterial strains from MALT lymphoma and those from other gastroduodenal diseases have been demonstrated. However, production of antibody to Hsp60 was less marked in other diseases with $H$ pylori infection. Undefined bacterial changes or host genetic backgrounds of immune responses to the bacterial infection may lead to enhanced immune responses to Hsp60 in patients with MALT lymphoma, but the reason for this specific response awaits clarification.

Induction of immune responses to host components may lead to tissue injury of an autoimmune nature. It is well known that gastric mucosal lesions of MALT lymphoma are often resistant to ordinary peptic ulcer treatment-that is, control of acid secretion. Immunological responses to $H$ pylori of both neoplastic B cells and non-neoplastic T cells have been reported in MALT lymphoma. ${ }^{18}$ In addition, gastric ulcers developed in severe combined immunodeficient mice transplanted with peripheral blood mononuclear cells from patients with MALT lymphoma when $H$ pylori was orally inoculated. ${ }^{19}$ These observations suggest that the development of gastric mucosal damage in patients with MALT lymphoma involves host immune responses to $H$ pylori. Hsp60 may be one of the target molecules.
In this study, a few $H$ pylori infected patients with gastric disease other than MALT lymphoma also had elevated IgG titres to HGC-27 cells and Hsp60. We are carefully following these patients to see whether they will develop gastric MALT lymphoma. In addition, the common association of anti-Hsp60 and anti-HGC-27 antibodies with MALT lymphoma suggests that measurement of these antibodies may provide a new diagnostic indicator for the disease.

Portions of this work were presented at the Xth International Workshop on Gastroduodenal Pathology and Helicobacter pylori and have been published in abstract form (Gut 1997;41(suppl 1):A63). The authors thank Dr William R Brown (University of Colorado School of Medicine) for assistance in preparation of the manuscript. This work was supported by a grant-in-aid for scientific research from the Japanese Ministry of Education, Science, Sports and Culture, Tokyo, Japan, and by the Mitsui Life Social Welfare Foundation.

1 Wotherspoon AC, Ortiz-Hidalgo C, Falzon MR, et al. Helicobacter pylori-associated gastritis and primary B-cell gastric lymphoma. Lancet 1991;338:1175-6.

2 Wotherspoon AC, Doglioni C, Diss TC, et al. Regression of primary low-grade B-cell gastric lymphoma of mucosaprimary low-grade B-cell gastric lymphoma of mucosabacter pylori. Lancet 1993;342:575-7.

3 Eidt S, Stole M, Fisher R. Helicobacter pylori gastritis and primary gastric non-Hodgkin's lymphoma. $\mathrm{f}$ Clin Pathol primary gastric

4 Bayerdorffer E, Neubauer A, Rudolph B, et al. Regression of primary gastric lymphoma of mucosa-associated lymphoid tissue type after cure of Helicobacter pylori infection. MALT Lymphoma Study Group. Lancet 1995;345:1591-4.

5 Negrini R, Savio A, Poiesi C, et al. Antigenic mimicry between Helicobacter pylori and gastric mucosa in the pathogenesis of body atrophic gastritis. Gastroenterology 1996;111:655-65.

6 Appelmelk BJ, Negrini R, Moran AP, et al. Molecular mimicry between Helicobacter pylori and the host. Trends Microbiol 1997;5:70-3.

7 Engstrand L, Scheynius A, Pahlson C. An increased number of gamma/delta T-cells and gastric epithelial cell expression of the groEL stress-protein homologue in Helicobacter pylori-associated chronic gastritis of the antrum. Am $f$ Gastroenterol 1991;86:976-80.

8 Harris NL, Jaffe ES, Stein H, et al. A revised EuropeanAmerican classification of lymphoid neoplasms: a proposal from the International Lymphoma Study Group. Blood 1994;84:1361-92.

9 Wotherspoon AC, Pan L, Diss TC, et al. A genotypic study of low grade B-cell lymphomas, including lymphomas of mucosa associated lymphoid tissue (MALT). F Pathol 1990;162:135-40

10 Yokota K, Oguma K, Yoshida H, et al. [Comparison of three methods for detecting Campylobacter pylori and measurement of human antibody titers against the whole organisms.] (In Japanese.) Kansenshogaku Zasshi 1990;64: 597-603.

11 Akagi T, Kimoto T. Human cell line (HGC-27) derived from the metastatic lymph node of gastric cancer. Acta Med Okayama 1976;30:215-19.

12 Macchia G, Massone A, Burroni D, et al. The Hsp60 of Helicobacter pylori: structure and immune response in patients with gastroduodenal diseases. Mol Microbiol 1993; 9:645-52.

13 Yokota K, Hirai H, Haque M, et al. Heat shock protein produced by Helicobacter pylori. Microbiol Immunol 1994;38: 403-5.

14 Kansau I, Labigne A. Heat shock proteins of Helicobacter pylori. Aliment Pharmacol Ther 1996;10:51-6.

15 Boog CJ, de Graeff-Meeder ER, Lucassen MA, et al. Two monoclonal antibodies generated against human hsp60 show reactivity with synovial membranes of patients with uvenile chronic arthritis. $\mathcal{F}$ Exp Med 1992;175:1805-10.

16 Kobayashi K, Yokota K, Kawahara Y, et al. Detection of the heat shock protein on the follicular dendritic cells in the germinal centers of low grade B cell gastric lymphoma of mucosa-associated lymphoid tissue (MALT) type. 7 Clin Pathol 1998;51:396-8.

17 Eck M, Schmausser B, Haas R, et al. MALT-type lymphoma of the stomach is associated with Helicobacter pylori of thains expressing theCagA protein. Gastroenterology 1997; 112:1482-6.

18 Hussell T, Isaacson PG, Crabtree JE, et al. The response of cells from low-grade B-cell gastric lymphomas of mucosaassociated lymphoid tissue to Helicobacter pylori. Lancet 1993;342:571-4.

19 Yokota K, Kobayashi K, Kawahara Y, et al. Gastric ulcers induced in MALToma hu-SCID mouse by oral infection of H. pylori [abstract]. Gut 1997;41:A119-20. 